\title{
SINGULAR INTEGRALS WITH POWER WEIGHTS
}

\author{
STEVE HOFMANN
}

(Communicated by J. Marshall Ash)

\begin{abstract}
This note contains proofs of weighted weak-type $(1,1)$ and weighted $L^{p}$ inequalities (with power weights $|x|^{\alpha}$ ) for singular integrals whose kernels satisfy Hörmander's condition, and also various size conditions. Some counter-examples are also given, yielding sharp results.
\end{abstract}

For singular integral operators in $\mathbf{R}^{n}$ defined by

$$
T f(x) \equiv \text { p.v. } \int K(x, y) f(y) d y,
$$

the weakest smoothness condition on the kernel $K$ known to yield a satisfactory $L^{1}$ theory is the so-called Hörmander condition:

$$
\int_{|x-y| \geq 2\left|y-y^{\prime}\right|}\left|K(x, y)-K\left(x, y^{\prime}\right)\right| d x \leq C .
$$

(Recently this has been relaxed in low dimensions, especially $n=2$. See [CR, $\mathrm{H} 1, \mathrm{H} 2])$. This smoothness condition is, however, too weak to prove weighted weak $(1,1)$ inequalities by any known method in the context of arbitrary $A_{1}$ weights, although for power weights $|x|^{\alpha}$ one $L^{1}$ result had been known in $\mathbf{R}^{n}$. Consider the case where $K$ is a homogeneous convolution kernel, i.e., $K(x)=\Omega(x)|x|^{-n}$ with $\Omega$ homogeneous of degree zero, integrable on the sphere and having mean value zero there. For such kernels, it is well known (see [CWZ, CZ]) that (1) is equivalent to the $L^{1}$-Dini Condition:

$$
\int_{0}^{1} \omega(t) t^{-1} d t<\infty, \quad \text { where } \omega(t) \equiv \sup \int_{|\sigma|=1}|\Omega(\rho \sigma)-\Omega(\sigma)| d \sigma,
$$

the sup running over all rotations $\rho$ with magnitude $|\rho| \leq t$. Kurtz and Wheeden [KW], have shown that, for $\Omega$ satisfying (2), the corresponding singular integral operator $T$ satisfies the weighted weak $(1,1)$ inequality

$$
\int_{\{|T f|>\lambda\}}|x|^{\alpha} d x \leq \frac{c}{\lambda} \int|f(x)||x|^{\alpha} d x
$$

if $-1<\alpha<0$. Also, the same authors give a counterexample of an $\Omega$ for which (2) holds, but (3) fails if $\alpha<-1$ (or $\alpha>0$.) It turns out that in fact more is

Received by the editors March 2, 1989.

1980 Mathematics Subject Classification (1985 Revision). Primary 42B20. 
true: by imposing a stronger size condition on $\Omega$, the range of $\alpha$ for which (3) holds can be expanded, even without strengthening the smoothness condition (2). Although not stated in [KW], for $\Omega \in L^{1}-\operatorname{Dini} \cap L^{q}(|x|=1), 1<q \leq \infty$, (3) holds if $-n+(n-1) / q<\alpha<0$, and furthermore this result could actually be obtained by modifying the argument in that paper, using a more general version of Lemma 1 of [KW] (see [MW, Lemma 1]). It is also possible, however, to give a simpler argument, and at the same time generalize the conditions on the kernel. For example, consider kernels $K(x, y)$ satisfying

$$
|K(x, y)| \leq A /|x-y|^{n} .
$$

For $p>1$, Stein [S] has shown that if $K$ satisfies (4), and if the corresponding operator $T$ is bounded on unweighted $L^{p}$, then $T$ is also bounded on $L^{p}\left(|x|^{\alpha} d x\right),-n<\alpha<n(p-1)$. For $p=1$, we have

Theorem 1. Let $T f(x) \equiv$ p.v. $\int K(x, y) f(y) d y$, where $T$ is bounded on (unweighted) $L^{2}$, and $K$ satisfies (1) and (4). Then the weighted weak $(1,1)$ bound (3) holds if $-n<\alpha<0$.

The unweighted $L^{2}$ bound is, of course, crucial. It is therefore worth noting that, for general nonconvolution kernels, it is an open problem to determine whether the Hörmander condition (1) (for both $K$ and its adjoint $K^{*}(x, y)=$ $K(y, x))$ is enough to imply a " $T 1$ " type criterion for $L^{2}$ boundedness. The weakest such smoothness condition known to yield a " $T 1$ " theorem is due to Meyer [M]. Meyer's condition is analogous to, but slightly stronger than (1), although it is still too weak to obtain $A_{1}$ weighted inequalities by any known method. Suppose that, for all $R>0,|u|+|v| \leq R$, and $k=1,2,3, \ldots$, that

$$
\int_{2^{k} R \leq|x-y| \leq 2^{k+1} R}|K(x, y)-K(x+u, y+v)| d x \leq \varepsilon(k) .
$$

Meyer's condition is that both $K$ and $K^{*}$ satisfy the above, and that

$$
\sum k \varepsilon(k)<\infty .
$$

It is well known that, for certain specific nonconvolution kernels, the $L^{2}$ bound can be obtained without Meyer's condition. One example is the commutator type operator with kernel

$$
K(x, y) \equiv \Omega(x-y)|x-y|^{-n-1}(a(x)-a(y)),
$$

where $\Omega$ is homogeneous of degree 0 , has first moment zero, and belongs to $L \log ^{+} L(|x|=1)$, and $\|\nabla a\|_{\infty}<\infty$ (see [C] or [BC]). If $\Omega$ also satisfies (2), then $K$ satisfies (1).

One can also prove (for a smaller range of $\alpha$ ) weighted inequalities with weaker size conditions than (4), although it seems that some sort of homogeneity must be assumed to obtain optimal results. In the unweighted case, $L^{p}$ and 
$w(1,1)$ bounds were proved by Benedek, Calderon, and Panzone [BCP] for convolution kernels satisfying

$$
\begin{gathered}
\left|\int_{0<\varepsilon \leq|x|<R<\infty} K(x) d x\right| \leq B \\
\int_{|x| \geq 2|y|}|K(x-y)-K(x)| d x \leq B
\end{gathered}
$$

and

$$
\int_{|x| \leq R}|x||K(x)| d x \leq B R .
$$

For homogeneous kernels, (5) is equivalent to $\Omega$ having mean value zero, (6) is equivalent to (2), and (7) is equivalent to $\Omega \in L^{1}$. As mentioned above, the operator $T$ induced by such a kernel satisfies the weighted $w(1,1)$ bound (3) if $-1<\alpha<0$ [KW]. Also, since $\Omega \in L^{1} \cap L^{1}$-Dini implies $\Omega \in L \log ^{+} L$ [CWZ], by [MW], $T$ is bounded on $L^{p}\left(|x|^{\alpha} d x\right),-1<\alpha<p-1$. In [MW] it is also shown that, for $\Omega \in L^{q}, q>1, T$ is bounded on $L^{p}\left(|x|^{\alpha} d x\right)$ if $\max \left(-n,-1-(n-1) p / q^{\prime}\right)<\alpha<\min \left(n(p-1), p-1+(n-1) p / q^{\prime}\right)$. The condition analogous to (7) corresponding to $\Omega \in L^{q}$ is

$$
\int_{|x| \leq R}|x|^{n q-n+1}|K(x)|^{q} d x \leq B R .
$$

Unfortunately, in this more general setting, the results of $[\mathrm{KW}]$ and $[\mathrm{MW}]$ need not hold.

Theorem 2. Let $1 \leq q<\infty$. For each $p \geq 1$, and each $\alpha<-n p / q^{\prime}$ or $\alpha>n p / q^{\prime}$, there exists a kernel $K(x)$ satisfying (5), (6), and (8), but for which the corresponding operator $T$ fails to be bounded on $L^{p}\left(|x|^{\alpha} d x\right), p>1$; if $p=1, T$ fails to satisfy the weighted $w(1,1)$ bound (3). In particular, for $q=1$ (condition (7)), the weighted bounds do not hold in general for any $\alpha \neq 0$.

If however, we assume a sort of average homogeneity, the standard results can be recovered. For $1 \leq q<\infty$, consider (not necessarily convolution) kernels for which both $K(x, y)$ and $K^{*}(x, y) \equiv K(y, x)$ satisfy

$$
\int_{a \leq|x-y| \leq b}|x-y|^{n q-n+1}|K(x, y)|^{q} d x \leq B(b-a) .
$$

Theorem 3. Suppose $T$ is bounded on unweighted $L^{p}, 1<p<\infty$, and suppose $K$ and $K^{*}$ satisfy Equation (9). Then $T$ is bounded on $L^{p}\left(|x|^{\alpha} d x\right)$ if $\max \left(-n,-1-(n-1) p / q^{\prime}\right)<\alpha<\min \left(n(p-1), p-1+(n-1) p / q^{\prime}\right)$. If $K$ also satisfies (1), then $T$ satisfies the $w(1,1)$ bound (3) for $-n+(n-1) / q<\alpha<0$. Remarks. For homogeneous convolution kernels, the $L^{p}$ result is that of [MW], and (for $q=1)$ the $w(1,1)$ inequality is that of $[\mathrm{KW}]$. For $q>1$, the weak $(1,1)$ result is new even for homogeneous convolution kernels, although in that special case the arguments of [KW] could have been modified as mentioned in the introduction of the present paper. 
By Theorem 2, the following result is sharp except for the end point values of $\alpha$ :

Theorem 4. Let $1<q<\infty$, and suppose $K$ and $K^{*}$ satisfy (9), but only for $a=0$ (i.e., this is the nonconvolution version of (8)). Suppose also that $T$ is bounded on unweighted $L^{p}(1<p<\infty)$. Then $T$ is bounded on $L^{p}\left(|x|^{\alpha} d x\right)$ if $\max \left(-n,-n p / q^{\prime}\right)<\alpha<\min \left(n(p-1), n p / q^{\prime}\right)$. Also, if $K$ satisfies (1), then the weak $(1,1)$ bound (3) holds for $-n / q^{\prime}=-n+n / q<\alpha<0$.

We now proceed to the proof of Theorem 1. Although easy and direct, it illustrates the basic set-up for all the $w(1,1)$ results in this paper.

The proof is a simple modification of Calderon-Zygmund arguments. For $\lambda>0$ fixed, perform a Whitney decomposition of the set $\{M f>\lambda\}$ (where $M$ is the Hardy-Littlewood Maximal operator) into a union of nonoverlapping closed cubes $Q_{j}$. We can write $f=b+g$, where $\int|g|^{2}|x|^{\alpha} d x \leq c \lambda \int|f||x|^{\alpha} d x$ and $b=\sum b_{j}$, with $b_{j}$ supported on $Q_{j}, \int b_{j}=0$, and $\int\left|b_{j}\right| \leq c \lambda\left|Q_{j}\right|$. One handles $g$ by the result of Stein [S] which implies that $T$ is bounded on $L^{2}\left(|x|^{\alpha} d x\right),-n<\alpha<n$.

Let $Q_{j}^{*}$ have the same center as $Q_{j}$, but side length $5 \sqrt{n}$ times as large (thus, for $x \in\left(Q_{j}^{*}\right)^{c}$ and $y \in Q_{j}$, one has $\left.|x-y| \geq 2 \operatorname{diam} Q_{j}\right)$, and let $E$ be the union of the cubes $Q_{j}^{*}$. By properties of $A_{1}$ weights, $\int_{E}|x|^{\alpha} d x \leq c \lambda^{-1} \int|f||x|^{\alpha} d x$, if $-n<\alpha<0$. Thus, as usual, it is enough to prove

$$
\int_{E^{c}}|T b(x)||x|^{\alpha} d x \leq c \int|b(y)||y|^{\alpha} d y .
$$

In fact, if $y_{j} \in Q_{j}$ is chosen so that $\left|y_{j}\right|^{\alpha}=\min _{Q_{j}}|y|^{\alpha}$, it is then enough to show

$$
\int_{\left(Q_{j}^{*}\right)^{c}}\left|T b_{j}(x)\right||x|^{\alpha} d x \leq c \int\left|b_{j}(y)\right|\left|y_{j}\right|^{\alpha} d y .
$$

The left-hand side of this last inequality is equal to

$$
\int_{|x| \leq\left|y_{j}\right| / 2}^{\left(Q_{i}^{*}\right)^{c}}+\int_{\substack{\left(Q_{j}^{*}\right)^{c} \\|x| y_{j} \mid / 2}} \equiv I_{1}+I_{2}
$$

Since $\alpha<0$, in $I_{2}$ we have $|x|^{\alpha} \leq c\left|y_{j}\right|^{\alpha}$, so $I_{2}$ is bounded by

$$
c \int_{\left(Q_{j}^{*}\right)^{c}}\left|\int b_{j}(y) K(x, y) d y\right|\left|y_{j}\right|^{\alpha} d x,
$$

which, as usual, by the mean value zero property of $b_{j}$ and Fubini's Theorem, is in turn no larger than

$$
c \int\left|b_{j}(y) \| y_{j}\right|^{\alpha} \int_{\left(Q_{j}^{*}\right)^{c}}\left|K(x, y)-K\left(x, y_{j}\right)\right| d x d y .
$$

Applying (1) and the definition of $Q_{j}^{*}$, we obtain the desired estimate. 
$I_{1}$ is also easy to handle. It is dominated by (11)

$$
\int\left|b_{j}(y)\right| \int_{|x| \leq\left|y_{j}\right| / 2}^{\left(Q_{j}^{*}\right)^{c}}|K(x, y)||x|^{\alpha} d x \leq C A \int\left|b_{j}(y)\right| \int_{|x| \leq\left|y_{j}\right| / 2}\left|y_{j}\right|^{-n}|x|^{\alpha} d x d y,
$$

where in the last step we have used (4) and also the fact that, for $x \in\left(Q_{j}^{*}\right)^{c}, y \in$ $Q_{j}$ and $|x| \leq\left|y_{j}\right| / 2$, we have $|x-y| \approx\left|y_{j}\right|$. Since $\alpha>-n$, the inner integral in the right-hand side of (11) is no bigger than $c\left|y_{j}\right|^{\alpha}$, which concludes the proof.

We remark that this result also holds for the maximal singular integral operator $\widetilde{T} f \equiv \sup _{\varepsilon>0}\left|T_{\varepsilon} f\right|$, where $T_{\varepsilon} f(x) \equiv \int_{|x-y|>\varepsilon} K(x, y) f(y) d y$. If $\widetilde{T}$ is bounded on unweighted $L^{2}$, then it is also bounded on $L^{2}\left(|x|^{\alpha} d x\right),-n<\alpha<$ $n$, by the results of [MW]. (That paper only discussed homogeneous kernels, but the same argument goes over unchanged for kernels which are merely bounded pointwise by a homogeneous kernel, i.e., $|K(x, y)| \leq|\Omega(x-y)| /|x-y|^{n}$, with $\Omega \in L^{q}\left(S^{n-1}\right)$. The case $q=\infty$ is the bound (4).) This takes care of the "good" function $g$. To handle the "bad" function $b$, we observe that, for each $x \in E^{c}$,

$$
\begin{aligned}
\tilde{T} b(x) & \leq \sup _{\varepsilon>0} \sum_{j} \chi_{j}(x)\left|T_{\varepsilon} b_{j}(x)\right| \\
& +\sup _{\varepsilon>0} \sum_{j}\left(1-\chi_{j}(x)\right)\left|T_{\varepsilon} b_{j}(x)\right| . \\
& \equiv A+B,
\end{aligned}
$$

where $\chi_{j}(x)$ is the characteristic function of $\left\{x \in E^{c}:|x| \leq\left|y_{j}\right| / 2\right\}$. Now

$$
A \leq \sum_{j} \chi_{j}(x) \int\left|K(x, y) \| b_{j}(y)\right| d y,
$$

which can be handled exactly like $I_{1}$ above by integrating over $E^{c}$ with respect to the measure $|x|^{\alpha} d x$. By exactly the same reasoning as in Stein's book [S2, pp. 43-44],

$$
B \leq \sum_{j}\left(1-\chi_{j}(x)\right) \int\left|K(x, y)-K\left(x, y_{j}\right)\right|\left|b_{j}(y)\right| d y+c M b(x),
$$

where $M$ is the Hardy-Littlewood maximal operator. This last sum is handled exactly like $I_{2}$ above by taking the weighted integral over $E^{c}$, and $M$ is well known to be $w(1,1)$ with respect to any $A_{1}$ weight.

We now proceed to the (simultaneous) proofs of Theorems 3 and 4, and defer the counter-examples of Theorem 2 until last. Let us assume for now the $L^{p}$ (or at least the $L^{2}$ ) results, and prove the weak $(1,1)$ bounds. We begin with the same decomposition as in the proof of Theorem 1, except that for technical reasons we take $Q_{j}^{*}$ to be large enough that, for $x \in\left(Q_{j}^{*}\right)^{c}$ and $y \in Q_{j}$, we have $|x-y| \geq 4 \operatorname{diam} Q_{j}$. Then, as before, modulo the $L^{2}$ result 
for the "good" function $g$, it is enough to prove inequality (10). The same splitting of the left side of (10) into $I_{1}+I_{2}$ is now used, with $I_{2}$ estimated exactly as in Theorem 1 (that estimate used only the Hörmander condition and the fact that $\alpha \leq 0)$. The estimate for $I_{1}$ imposes the lower bound on $\alpha$, and is the only new difficulty here. Again, it is enough to show that

$$
\int_{\substack{\left.\mid Q_{j}^{*}\right)^{c} \\\left(Q_{j}^{*} \mid / 2\right.}}|K(x, y)||x|^{\alpha} d x \leq C|y|^{\alpha},
$$

for all $y \in Q_{j}$ and $\alpha>-n+(n-1) / q \quad(1 \leq q<\infty$, if (9) holds as in Theorem $3)$ or $\alpha>-n+n / q(q>1$, if (9) only holds for $a=0$ as in Theorem 4). Note that if $\left|y_{j}\right| \leq 2 \operatorname{diam} Q_{j}$, then the left side of (12) is zero, because in that case $|x| \geq\left|x-y_{j}\right|-\left|y_{j}\right| \geq 4 \operatorname{diam} Q_{j}-2 \operatorname{diam} Q_{j} \geq\left|y_{j}\right|$. Thus we make take $\left|y_{j}\right|>2 \operatorname{diam} Q_{j}$, so, for all $y \in Q_{j},|y| \approx\left|y_{j}\right| \approx|x-y|$. The left side of (12) is therefore bounded by

$$
\int_{a|y| \leq|x-y| \leq b|y|}|K(x, y)||x|^{\alpha} d x
$$

where $0<a<b<\infty$ and $a, b$ depend only on dimension. Theorem 4 ( $p=1$ case) now follows directly from Hölder's inequality. In fact, for $\alpha>-n+n / q$ (i.e., $\left.a q^{\prime}>-n\right),(13)$ is less than or equal to

$$
\left(\int_{a|y| \leq|x-y| \leq b|y|}|K(x, y)|^{q} d x\right)^{1 / q}\left(\int_{|x| \leq(b+1)|y|}|x|^{\alpha q^{\prime}} d x\right)^{1 / q^{\prime}} .
$$

By the assumption on $\alpha$, the second factor equals $C|y|^{\alpha+n / q^{\prime}}$. The first factor is no larger than

$$
C\left(|y|^{-q n+n-1} \int_{a|y| \leq|x-y| \leq b|y|}|K(x, y)|^{q}|x-y|^{q n-n+1} d x\right)^{1 / q} \leq c|y|^{-n+n / q} .
$$

Multiplying then gives the desired estimate.

The $w(1,1)$ part of Theorem 3 will be an easy consequence of

Lemma 1. Let $K$ satisfy (9), and let $0<\gamma<1$. Then

$$
\int_{a|y| \leq|x-y| \leq b|y|}|x|^{-\gamma}|K(x, y)|^{q} d x \leq C|y|^{-n q+n-\gamma} .
$$

Proof. Assume for now that Lemma 1 holds. If $q=1$, set $-\gamma=\alpha$ and we are done. For $q>1$, and $\alpha>-n+(n-1) / q$, choose $\varepsilon>0$ so that $\alpha>$ $-n+(n-1) / q+\varepsilon$, and set $\beta=1 / q-\varepsilon$ (thus $\beta q<1$ ). Write $|x|^{\alpha}=|x|^{-\beta}|x|^{\alpha+\beta}$ and apply Hölder's inequality to (13), which is then dominated by

$$
\left(\int_{a|y| \leq|x-y| \leq b|y|}|K(x, y)|^{q}|x|^{-\beta q} d x\right)^{1 / q}\left(\int_{|x| \leq(b+1)|y|}|x|^{(\alpha+\beta) q^{\prime}} d x\right)^{1 / q^{\prime}} .
$$


By (14), with $\gamma=\beta q$, the first factor is less than or equal to $c|y|^{-n+n / q-\beta}$. The second factor equals $c|y|^{n / q^{\prime}+\alpha+\beta}$, since the definition of $\beta$ and the assumptions on $\alpha$ and $\varepsilon$ imply that $(\alpha+\beta) q^{\prime}>-n$. The estimate (12) then follows, so it is enough to prove Lemma 1 .

We remark that, for convolution kernels, Lemma 1 is very easy to prove: For $-\gamma<0$, we can replace $|x|$ by ||$x-y|-| y||$ in the left side of (14), write the integral in polar coordinates centered at $y$, and use the fact that, by Lebesque's differentation theorem, inequality (9) for convolution kernels is equivalent to $r^{n q} \int_{S^{n-1}}|K(r \sigma)|^{q} d \sigma \leq B$ for a.e. $r \in(0, \infty)$.

To prove (14) in the general case, we split the integral into

$$
\int_{a|y| \leq|x-y| \leq|y|}|x|^{-\gamma}|K(x, y)|^{q} d x+\int_{|y| \leq|x-y| \leq b|y|}|x|^{-\gamma}|K(x, y)|^{q} d x .
$$

We estimate only the second term (the first is handled analogously), and dominate it by

$$
\sum_{k=1}^{\infty} \int_{A_{k}}|K(x, y)|^{q}|| x-y|-| y \|^{-\gamma} d x,
$$

where $A_{k}=\left\{x:|y|\left(1+(b-1) 2^{-k}\right) \leq|x-y| \leq|y|\left(1+(b-1) 2^{-k+1}\right)\right\}$. Now $|x-y| \approx|y|$ (independently of $k$ ), and, on $A_{k},|x-y|-|y| \approx|y| 2^{-k}$. Thus, (15) is bounded by

$$
\begin{aligned}
& C \sum_{k=1}^{\infty}|y|^{-\gamma} 2^{k \gamma}|y|^{-q n+n-1} \int_{A_{k}}|x-y|^{q n-n+1}|K(x, y)|^{q} d x \\
& \quad \leq C|y|^{-q n+n-\gamma} \sum_{k=1}^{\infty} 2^{k(\gamma-1)},
\end{aligned}
$$

where in the last inequality we have applied (9) to the integral over $A_{k}$. For $\gamma<1$, (14) now follows.

Next we prove the $L^{p} \quad(p>1)$ bounds of Theorems 3 and 4 . The proof essentially follows that of [MW], the only modification being to use the techniques already discussed in the $w(1,1)$ case. The argument will therefore be kept brief. As in [MW], it is enough to prove weighted $L^{p}$ bounds for

$$
R f(x) \equiv \int_{|y| \leq|x| / 2}|K(x, y)||f(y)| d y
$$

and

$$
S f(x) \equiv \int_{|y| \geq 2|x|}|K(x, y) f(y)| d y .
$$

The upper limit for $\alpha$ comes from the estimate for $R f$, the lower limit from $S f$. (The remaining part of the operator, corresponding to (4.2) on page 255 of [MW], is handled exactly as in that paper. The $L^{p}$ bound for this part depends only on the unweighted $L^{p}$ bound for $T$, and imposes no restriction on $\alpha$.) In fact, it is enough to study $R f$, because the estimate for $S f$ then follows 
by duality as in Lemma 5 of [MW]. Let $m=\min (1, q / p)$. Set $\beta=\alpha / p$. Following the proof of Lemma 3 of [MW], we apply Hölder's inequality and bound $|x|^{\beta} R f(x)$ by the product of

$$
\left(\int_{|y| \leq|x| / 2}\left(|f(y)||y|^{\beta+\varepsilon}|K(x, y)|^{m}\right)^{p} d y\right)^{1 / p} \equiv P(x)
$$

and

$$
|x|^{\beta}\left(\int_{|y| \leq|x| / 2}\left(|y|^{-\beta-\varepsilon}|K(x, y)|^{1-m}\right)^{p^{\prime}} d y\right)^{1 / p^{\prime}} \equiv|x|^{\beta} Q(x),
$$

where $\varepsilon$ is a small positive number to be chosen. If $m=1$ (i.e., $q \geq p$ ), then $|x|^{\beta} Q(x)$ is bounded by $C|x|^{n / p^{\prime}-\varepsilon}$, if $\varepsilon$ is chosen small enough so that $p^{\prime}(\beta+\varepsilon)<n$, which of course can always be done if $\alpha<n(p-1)$. If $q<p$, then $m=q / p$ and $(1-m) p^{\prime}=(p-q) /(p-1)$. Set $\delta=q(p-1) /(p-q)$ (which is bigger than 1 for $q>1)$. If $q=1=\delta$, and $K^{*}$ satisfies (9) as in Theorem 3 , then Lemma 1 with $\gamma=p^{\prime}(\beta+\varepsilon)$ can be applied directly to obtain the bound $|x|^{\beta} Q(x) \leq C|x|^{-\varepsilon}$, for $\beta+\varepsilon<1 / p^{\prime}=(p-1) / p$. Now, for $1<q<p$, apply Hölder's inequality again, so that

$$
\begin{aligned}
Q(x)^{p^{\prime}} \leq & \left(\int_{|x| / 2 \leq|x-y| \leq 3|x| / 2}|K(x, y)|^{q}|y|^{-\eta \delta} d y\right)^{1 / \delta} \\
& \times\left(\int_{|y| \leq|x| / 2}|y|^{\delta^{\prime}\left(\eta-p^{\prime}(\beta+\varepsilon)\right)} d y\right)^{1 / \delta^{\prime}},
\end{aligned}
$$

where $\eta=1 / \delta-\varepsilon$, so $\eta \delta=1-\delta \varepsilon<1$. Applying Lemma 1 with $\eta \delta=\gamma$, we see that the first factor in (16) is bounded by

$$
C|x|^{-\eta-n(q-1) / \delta} .
$$

The second factor is no larger than

$$
C|x|^{\eta-p^{\prime}(\beta+\varepsilon)+n / \delta^{\prime}}
$$

if $\delta^{\prime}\left(\eta-p^{\prime}(\beta+\varepsilon)\right)>-n$. But a grubby computation shows that this is true if $\beta<(n-1) / q^{\prime}+1 / p^{\prime}-\varepsilon\left(1+1 / p^{\prime}\right)$, which holds under the assumptions of Theorem 3, for $\varepsilon$ small enough. For Theorem 4, we obtain the same estimates that $\eta=0$, since $\beta<n / q^{\prime}$ implies $\delta^{\prime} p^{\prime}(\beta+\varepsilon)<n$ for small $\varepsilon$. In any case multiplying these estimates, taking the power $1 / p^{\prime}$, and multiplying by $|x|^{\beta}$ shows that

$$
|x|^{\beta} Q(x) \leq|x|^{n / p^{\prime}-n q / \delta p^{\prime}-\varepsilon}=|x|^{-\varepsilon+n(q-1) / p} .
$$

Now we multiply $P(x)$ and the estimate for $|x|^{\beta} Q(x)$, so that, in the case 
$q<p(m=q / p)$, we have

$$
\begin{aligned}
& \int\left(|x|^{\beta} R f(x)\right)^{p} d x \\
& \quad \leq C \iint_{|y| \leq|x| / 2}|f(y)|^{p}|y|^{\beta p+\varepsilon p}|K(x, y)|^{q} d y|x|^{-\varepsilon p+n q-n} d x \\
& \quad=\int|f(y)|^{p}|y|^{\alpha} \int_{|y| \leq|x| / 2}(|y| /|x|)^{\varepsilon p}|K(x, y)|^{q}|x|^{q n-n} d x d y .
\end{aligned}
$$

The inner integral equals

$$
\begin{aligned}
& |y|^{p \varepsilon} \int_{|x| \geq 2|y|}|x|^{-\varepsilon p-1}|K(x, y)|^{q}|x|^{q n-n+1} d x \\
& \quad \leq C|y|^{p \varepsilon} \sum_{k=1}^{\infty}|y|^{-p \varepsilon-1} 2^{k(-p \varepsilon-1)} \int_{2^{k}|y| \leq|x| \leq 2^{k+1}|y|}|K(x, y)|^{q}|x-y|^{q n-n+1} d x \\
& \quad \leq C \sum_{k=1}^{\infty} 2^{-k p \varepsilon}\left(2^{k}|y|\right)^{-1} \int_{|x-y| \leq 2^{k+2}|y|}|K(x, y)|^{q}|x-y|^{q n-n+1} d x \leq C B
\end{aligned}
$$

by (9) applied with $a=0$.

To conclude the proofs of Theorems 3 and 4 , we consider the case $q \geq p$ $(m=1)$. In this case we had the bound $|x|^{\beta} Q(x) \leq c|x|^{n / p^{\prime}-\varepsilon}$, so

$$
\begin{aligned}
& \int\left(|x|^{\beta} R f(x)\right)^{p} d x \\
& \quad \leq \int|f(y)|^{p}|y|^{\beta p} \int_{|x| \geq 2|y|}|K(x, y)|^{p}(|y| /|x|)^{\varepsilon p}|x|^{n p-n} d x d y .
\end{aligned}
$$

But the inner integral is bounded by a constant just as in the previous case, because if (9) is satisfied for a given $q>1$, it also is satisfied for $p<q$ by Hölder's inequality.

We remark that these $L^{p}$ results also carry over for the maximal singular integral $\widetilde{T}$, if $\widetilde{T}$ is known to be bounded on unweighted $L^{p}$. The operators $R$ and $S$ are clearly bounded independently of any truncation of the kernel, and the rest of the operator $\widetilde{T}$ is exactly like (4.2) of [MW, p. 255].

It remains only to discuss the counterexamples of Theorem 2 . For simplicity, we give an example for the case $q=1=p$, followed by a brief sketch of the modifications necessary for the other cases.

Set $\overline{1}=(1,0,0, \ldots, 0)$, and, for $0<\gamma<1$, define

$$
K(x) \equiv \begin{cases}|x+\overline{1}|^{-n+\gamma}, & \text { if }|x+\overline{1}| \leq \frac{1}{2} \\ -|x-\overline{1}|^{-n+\gamma}, & \text { if }|x-\overline{1}| \leq \frac{1}{2} \\ 0, & \text { otherwise }\end{cases}
$$

and, for $N=4,5,6, \ldots$,

$$
f_{N}(y) \equiv \text { characteristic function of }\{|y-\overline{1}| \leq 1 / N\} .
$$


Then $\int f_{N}(y)|y|^{\alpha} d y \approx C N^{-n}$. Note that $K$ trivially satisfies (6) and (7), because it is integrable, and (5) because it is odd. Now for $|x| \leq 1 / N$ and $|y-\overline{1}| \leq 1 / N$, we have $|x-y+\overline{1}|=|y-x-\overline{1}| \leq 2 / N$. Thus, for such $x$,

$$
\begin{aligned}
K^{*} f_{N}(x) & =\int_{|y-\overline{1}| \leq 1 / N}|x-y+\overline{1}|^{-n+\gamma} d y \\
& \geq C\left(\frac{1}{N}\right)^{-n+\gamma} \int_{|y-\overline{1}| \leq 1 / N} d y \\
& =C N^{n-\gamma}\left(\frac{1}{N}\right)^{n}=C N^{-\gamma} .
\end{aligned}
$$

Observe that

$$
C \int_{|x| \leq 1 / N}|x|^{\alpha} d x=\left(\frac{1}{N}\right)^{n+\alpha}=N^{-n-\alpha}, \quad \alpha>-n .
$$

If $T f \equiv K^{*} f$ were of weak-type $(1,1)$ with respect to $|x|^{\alpha}$, we would have

$$
\begin{aligned}
N^{-n-\alpha} & \leq \int \chi\left\{\left|T f_{N}\right|>c N^{-\gamma}\right\}|x|^{\alpha} d x \\
& \leq c N^{\gamma} \int f_{N}(y)|y|^{\alpha} d y \\
& \leq c N^{-n+\gamma} .
\end{aligned}
$$

But letting $N \rightarrow \infty$, we obtain a contradiction if $\alpha<-\gamma$. Since $\gamma$ could be chosen arbitrarily close to zero, we are done.

The remaining cases of Theorem 2 are easy variants of the preceeding. For $q>1$, use the kernel $K_{q} \equiv K^{1 / q}$, where $K$ is as above. The $w(1,1)$ and also the $L^{p}, p>1$ arguments for $\alpha<0$ are then handled like the above. The upper limit for $\alpha$ with $p>1$ is obtained by duality.

\section{REFERENCES}

[BC] B. Bajsanski and R. Coifman, On singular integrals, Proc. Sympos. Pure Math. 10 (1967), $1-17$.

[BCP] A. Benedek, A. P. Calderon, and R. Panzone, Convolution operators on Banach space valued functions, Proc. Nat. Acad. Sci. 48 (1962), 356-365.

[C] A. P. Calderon, Commutators of singular integral operators, Proc. Nat. Acad. Sci. 53 (1965), 1092-1099.

[CWZ] A. P. Calderon, M. Weiss, and A. Zygmund, On the existence of singular integrals, Proc. Sympos. Pure Math. 10 (1967), 56-73.

[CZ] A. P. Calderon and A. Zygmund, A note on singular integrals, Studia Math. 65 (1979), 77-87.

[CR] M. Christ and J. L. Rudio de Francia, Weak-type $(1,1)$ bounds for rough operators II, Invent. Math. 93 (1988), 225-237.

[H1] S. Hofmann, Weak $(1,1)$ boundedness of singular integrals with nonsmooth kernel, Proc. Amer. Math. Soc. 103 (1988), 260-264.

[H2] - Weighted weak-type $(1,1)$ bounds for rough operators, preprint.

[KW] D. S. Kurtz and R. L. Wheeden, A note on singular integrals with weights, Proc. Amer. Math. Soc. 81 (1981), 391-397.

[M] Y. Meyer, La continuite des operateurs definis par des integrales singulieres, preprint. 
[MW] B. Muckenhoupt and R. L. Wheeden, Weighted norm inequalities for singular and fractional integrals, Trans. Amer. Math. Soc. 161 (1971), 249-258.

[S] E. M. Stein, Note on singular integrals, Proc. Amer. Math. Soc. 8 (1957), 250-254.

[S2] _ Singular integrals and differentiability properties of functions, Princeton Univ. Press, Princeton, 1970.

Department of Mathematics and Statistics, McMaster University, Hamilton, ONTARIO L8S 4K1, Canada 